\title{
Cut-C: cleavage under tethered nuclease for conformational capture
}

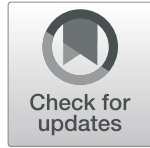

Takashi Shimbo $^{1^{*}}$ D, Machika Kawamura ${ }^{1,2}$, Edward Wijaya ${ }^{1,2}$, Eiichi Takaki ${ }^{1,2}$, Yasufumi Kaneda $^{3}$ and Katsuto Tamai ${ }^{{ }^{*}}$

\begin{abstract}
Background: Deciphering the 3D structure of the genome is essential for elucidating the regulatory mechanisms of gene expression in detail. Existing methods, such as chromosome conformation capture (3C) and Hi-C have enabled the identification of novel aspects of chromatin structure. Further identification of protein-centric chromatin conformation is enabled by coupling the $\mathrm{Hi}-\mathrm{C}$ procedure with a conventional chromatin immunoprecipitation assay. However, these methods are time-consuming and require independent methods for validation.

Results: To simultaneously identify protein-centric chromatin conformation and target protein localization, we have developed Cut-C, a method that combines antibody-mediated cleavage by tethered nuclease with chromosome conformation capture to identify chromatin interactions mediated by a protein of interest. Applying Cut- $C$ to H3K4me3, a histone modification enriched at active gene promoters, we have successfully identified chromatin loops mediated by H3K4me3 along with the genome-wide distribution of H3K4me3. Cut-C also identified chromatin loops mediated by CTCF, validating the general applicability of the method.

Conclusions: Cut-C identifies protein-centric chromatin conformations along with the genome-wide distribution of target proteins using simple procedures. The simplified protocol will improve the efficiency of analysing chromatin conformation using precious materials, such as clinical samples.
\end{abstract}

Keywords: Chromosome conformation, Cut-C, Gene regulation, Next-generation sequencing

\section{Background}

Resolution of the three dimensional (3D) conformation of chromatin is essential for understanding the regulatory mechanisms involved in gene expression [1]. Chromatin conformation signatures determine the lineage-specific differentiation of cells with identical genomes. Understanding how multiple proteins, such as CTCF, regulate chromatin conformation is essential to fully appreciate the complexity of gene expression regulation [2].

Revolutionary techniques, such as chromosome conformation capture (3C) and its sequencing version, $\mathrm{Hi}-\mathrm{C}$, have enabled the understanding of numerous aspects of chromatin conformation, including gene loops, promoter-enhancer loops, and topologically associated domains [3, 4]. Further derivatives of $\mathrm{Hi}-\mathrm{C}$,

\footnotetext{
*Correspondence: shimbot@sts.med.osaka-u.ac.jp; tamai@gts.med.osakau.ac.jp

${ }^{1}$ Department of Stem Cell Therapy Science, Graduate School of Medicine, Osaka University, Suita, Osaka 5650871, Japan

Full list of author information is available at the end of the article
}

such as ChIA-PET and HiChIP, have been developed to analyse the protein-centric chromatin conformation by coupling the $\mathrm{Hi}-\mathrm{C}$ procedure with the conventional chromatin immunoprecipitation assay (ChIP) [5-7]. Genome wide localization information of a target protein along with the chromatin conformation mediated by the protein is essential to uncover protein-centric chromatin conformation $[8,9]$. However, because these techniques are mainly focused on identifying chromatin conformation, independent ChIP-seq experiments (or equivalents), which are generally time-consuming, need to be performed to precisely map the genome wide localization of target proteins. Therefore, an improved technique to simultaneously identify the chromatin conformation and precisely map target proteins using simple and robust procedures is essential. In this study, we aimed to develop a method that combines antibody-mediated cleavage by tethered nuclease with chromosome conformation capture to identify chromatin interactions mediated 
by a protein of interest along with the genome-wide distribution of the target proteins.

\section{Results and discussion}

To simultaneously identify the chromatin conformation and precisely map target proteins, we have developed $\mathrm{Cut}-\mathrm{C}$, a method implementing the cleavage under tethered nuclease technology into Micro- $\mathrm{C}$, a $\mathrm{Hi}-\mathrm{C}$ derivative using micrococcal nuclease (MNase) instead of restriction enzymes, to fragment chromatin $[10,11]$. In $\mathrm{Hi}-\mathrm{C}$ derivatives, DNA interactions are labelled by specific cleavages (mediated by restriction enzymes, mechanical forces, or MNase) followed by proximity ligation. In contrast, in Cut-C, only a subset of the long-range DNA interactions, whose conformation is mediated by a target protein, is labelled by a spatially controlled cleavage by protein A-fused MNase, which is tethered by antibodies against a target protein, followed by proximity ligation and subsequent DNA interaction enrichment and amplification by a tagmentation-based library preparation method. The resulting libraries can be analysed by massive parallel sequencing in a pairedend mode. Similar to other $\mathrm{Hi}-\mathrm{C}$ derivatives, pairedend tags (PETs) yield chromatin conformation data. Furthermore, as the cleavage of MNase is tightly regulated at the protein binding location, identification of cleaved sites provides a precise map for the target protein. The simple Cut- $\mathrm{C}$ experiment can be conducted in 3 days (Fig. 1a and Methods).

To test this method, we performed Cut-C for active histone marks using anti-H3K4me3 antibody in a commonly used human cell line, HEK293T (Additional file 1). To assess reproducibility, we prepared Cut-C libraries with two biological replicates (10 million cells) and obtained 206,471,443 and 139,158,639 paired-end sequencing reads, respectively. We first assessed the power of Cut-C to identify the genome wide localization of a target protein; we analysed Cut- $\mathrm{C}$ data (paired-end data were decoupled and analysed as single-end data, in general) with standard ChIP-seq analysis pipeline. To evaluate the localization identified by $\mathrm{Cut}-\mathrm{C}$, we performed CUT\&RUN with anti-H3K4me3 antibody in HEK293T cells. A representative screenshot of the genome browser

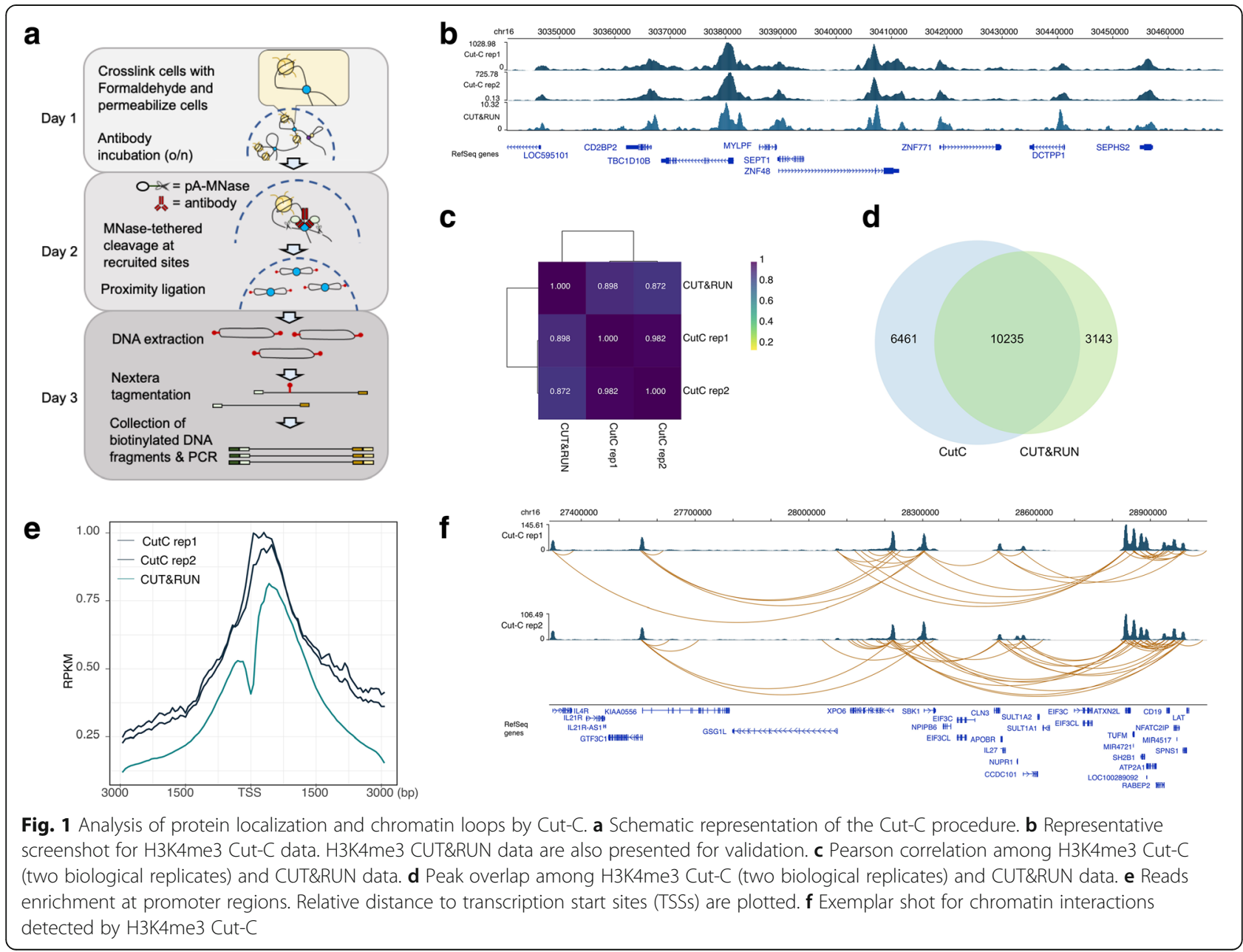


shows that Cut-C provides protein localization information with high quality in good agreement with the CUT\&RUN data (Fig. 1b) with high overall correlation (Fig. 1c). To further validate the enrichment specificity, ChIP peaks were called using MACS2. We identified 16, 696 peaks with Cut-C data (two replicates combined) and 13,378 peaks using CUT\&RUN data, and the identified peaks show a high degree of overlap (>61\%) (Fig. $1 d)$. We also confirmed that the Cut-C reads were strongly enriched around transcription start sites (TSSs), reflecting the H3K4me3 modification pattern (Fig. 1e), suggesting that Cut-C can provide protein localization information.

To assess the chromatin interactions identified by Cut-C, we analysed the data using the HiC-Pro pipeline [12]. Approximately $59 \%$ of the total sequencing reads were informative and valid paired end tags (PETs), as defined by HiC-Pro in the Cut-C data for H3K4me3 in HEK293T cells (Additional file 2). We detected 206,471,443 and 139,158, 639 total informative PETs in the two biological replicates, respectively. Of those, majority of the identified PETs was intra-chromosomal PETs and only about $5 \%$ was consisted by the inter-chromosomal PETs (Additional file 2). Although the intra-chromosomal PETs showed high consistency between the biological replicates (more than $90 \%$ overlap), but only about $13 \%$ of the inter-chromosomal PETs were overlapped (Additional file 3). Further investigations will be required to judge whether these interchromosomal PETs are bone fide interactions. Using the cLoop pipeline, we call loops using the identified PETs by Cut-C. We detected 98,199 (H3K4me3 replicate 1) and 131,508 (H3K4me3 replicate 2) loops (Additional file 2). The example screenshot shows the chromatin loops identified using Cut-C (Fig. 1f). The genomic distance of the identified loops showed agreement between biological replicates, validating the reproducibility of the Cut-C procedure (Additional file 4).

To test general applicability, we performed Cut-C using an anti-CTCF antibody in HEK293T cells (10 million cells) and obtained 140,345,513 sequencing reads and 52,518 loops (Additional file 2). CTCF Cut-C successfully identified the chromatin loops (Fig. 2a). The example genome browser shot and Pearson correlation showed a high agreement between Cut-C and CTCF CUT\&RUN data (Fig. 2a and b). We identified 23,145 and 15,766 peaks using Cut-C and CUT\&RUN data, respectively, and the called peak showed a high degree of overlap (Fig. 2c). We also confirmed that Cut-C recapitulated the previously identified orientation of the CTCF motif (Fig. 2d), underlining its applicability to multiple antibodies.

Using the targeted cleavage of the antibody-tethered MNase, we successfully eliminated the ChIP step, which is time-consuming and laborious. Most methods to identify protein-centric chromatin conformation, including

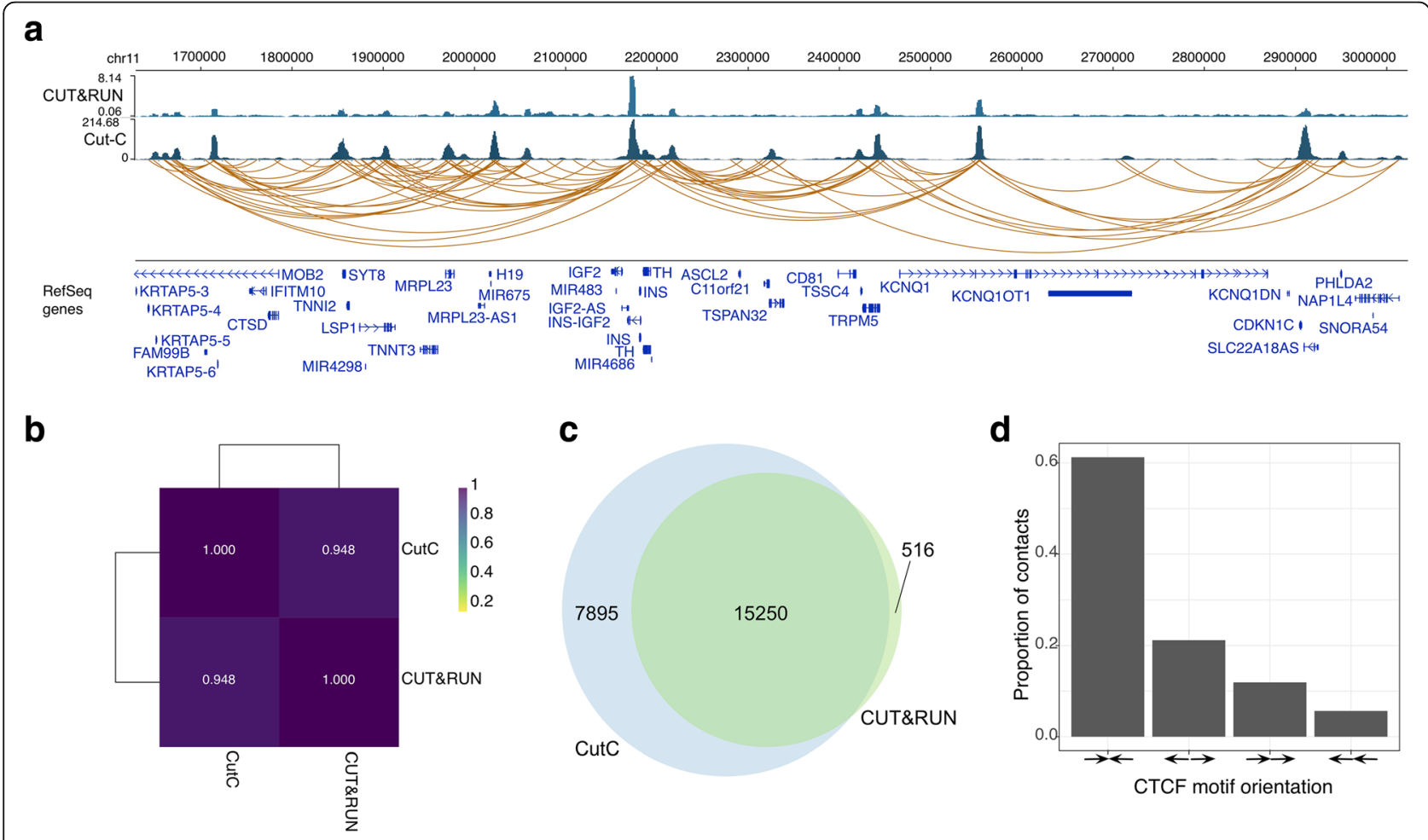

Fig. 2 General applicability of Cut-C. a Exemplar shot for CTCF Cut-C. b Pearson correlation between CTCF Cut-C and CUT\&RUN data. c Peak overlap between CTCF Cut-C and CUT\&RUN data. $\mathbf{d}$ CTCF motif orientation in read pairs 
HiChIP, involve ChIP to enrich the target protein. Because Cut-C does not require chromatin solubilization, we could omit chromatin fragmentation by sonication, which potentially denatures epitopes [13].

\section{Conclusions}

Here we introduced Cut-C, a simple method to delineate the protein-centric chromatin conformation and protein localization using a single experiment. By targeting H3K4me3 and CTCF, we showed that Cut-C has the potential to detect chromatin conformation. The simplified procedure of Cut-C will also facilitate the analysis of chromatin conformation in various biological samples, including patient clinical samples.

\section{Methods}

\section{Cell culture and sampling}

HEK293T cells were cultured in 10\% FBS/ 1\% penicillinstreptomycin/DMEM. Cells were trypsinized and washed with $2 \% \mathrm{FBS} / \mathrm{PBS}$ and $1 \times 10^{7}$ cells were centrifuged at $300 \times g$ for $5 \mathrm{~min}$ at room temperature. Cells were fixed with $1 \%$ formaldehyde/2\% FBS/PBS $(1 \mathrm{ml})$ for $10 \mathrm{~min}$ at room temperature. Fixation was quenched with ice-cold glycine to a final concentration of $250 \mathrm{mM}$ for $5 \mathrm{~min}$ at room temperature with gentle rocking, and on ice for 15 min with occasional mixing. Cells were centrifuged at $800 \times g$, $5 \mathrm{~min}, 4^{\circ} \mathrm{C}$; washed twice with PBS; and snap-frozen with liquid nitrogen.

\section{Cut-C}

Cells were first lysed with lysis buffer $(10 \mathrm{mM}$ Tris$\mathrm{HCl}$ (pH 7.4), $10 \mathrm{mM} \quad \mathrm{NaCl}, 3 \mathrm{mM} \quad \mathrm{MgCl}_{2}, \quad 0.1 \%$ Tween 20, 0.1\% IGEPAL CA-630, 0.01\% Digitonin) for $30 \mathrm{~min}$ on ice, with occasional mixing [14-16]. Lysis was stopped by adding an equal volume of resuspension buffer (10 mM Tris- $\mathrm{HCl}$ (pH 7.4), $10 \mathrm{mM}$ $\mathrm{NaCl}, 3 \mathrm{mM} \mathrm{MgCl}_{2}, 0.1 \%$ Tween 20), and the cell pellet was collected by centrifugation $(800 \times g)$ for 5 min at $4{ }^{\circ} \mathrm{C}$. The cell pellet was washed twice with wash buffer (20 mM HEPES, $150 \mathrm{mM} \mathrm{NaCl}, 0.5 \mathrm{mM}$ Spermidine, $1 \times$ Protease Inhibitor) $[11,16]$ and was resuspended in $500 \mu \mathrm{L}$ antibody-containing buffer (20 mM HEPES, $150 \mathrm{mM} \mathrm{NaCl}, 0.5 \mathrm{mM}$ Spermidine, $1 \times$ Protease Inhibitor, $0.02 \%$ or $0.0375 \%$ Digitonin, 2 $\mathrm{mM}$ EDTA). Cells were incubated overnight with $1 \mu \mathrm{g}$ anti- H3K4me3 antibody (Abcam; ab8580) or $5 \mu \mathrm{g}$ anti-CTCF antibody (Abcam; ab70303), followed by centrifugation at $500 \times g$ for $5 \mathrm{~min}$ at $4{ }^{\circ} \mathrm{C}$ and washed twice with $1 \mathrm{ml}$ digitonin buffer $(20 \mathrm{mM}$ HEPES, $150 \mathrm{mM} \mathrm{NaCl}, \quad 0.5 \mathrm{mM}$ Spermidine, $1 \times$ Protease Inhibitor, $0.02 \%$ or $0.0375 \%$ Digitonin). The cell pellet was resuspended with digitonin buffer and incubated with empirically tested Protein A-MNase (Protein A-MNase fused protein was purified as previously described with minor modifications [17]) for $1 \mathrm{~h}$ at $4{ }^{\circ} \mathrm{C}$ with rotation, as previously described. MNase-tethered cleavage was performed by incubating the sample at $37^{\circ} \mathrm{C}$ for $20 \mathrm{~min}$. The CUT\&RUN was stopped by adding EGTA to a final concentration of $2 \mathrm{mM}$ and incubated at $65^{\circ} \mathrm{C}$ for $10 \mathrm{~min}$. The cell pellet was processed for dephosphorylation, endchewing, end-labelling, and in situ proximity ligation of DNA fragments cleaved by tethered protein AMNase as previously described, with minor modifications [18].

\section{Library preparation}

Genomic DNA samples from Cut-C were purified through phenol-chloroform extraction and ethanol precipitation [18]. After air-drying, the pellet was resuspended in $50 \mu \mathrm{L}$ Buffer EB (Qiagen) and heated at $37^{\circ} \mathrm{C}$ for $15 \mathrm{~min}$. The sample was then purified further using Genomic DNA Clean \& Concentrator (Zymo Research) and eluted in $50 \mu \mathrm{L}$ Buffer EB (Qiagen). The concentration of DNA was measured with Qubit 3.0 Fluorometer (Invitrogen/Life Technologies). Multiple $50 \mu \mathrm{L}$ aliquots, consisting of $1 \mu \mathrm{L}$ Tagment DNA Enzyme 1(Nextera DNA Library Prep Kit, Illumina), 21.5 ng or 10 ng DNA and $25 \mu \mathrm{L}$ Tagment DNA Buffer (Nextera DNA Library Prep Kit, Illumina), were prepared for tagmentation. Tagmentation was performed at $55^{\circ} \mathrm{C}$ for $10 \mathrm{~min}$. DNA was extracted using Genomic DNA Clean \& Concentrator (Zymo Research) and eluted with $50 \mu \mathrm{L}$ Buffer EB (Qiagen). Ten microliters Dynabeads M-280 Streptavidin beads (Thermo Fisher Scientific) were washed and prepared. Briefly, the $50 \mu \mathrm{L}$ chromatin sample and $50 \mu \mathrm{L}$ bead suspension were mixed and incubated for $30 \mathrm{~min}$ for room temperature on a rotor. The biotin-tagged DNA fragments were collected on a magnetic stand and resuspended in $20 \mu \mathrm{L}$ or $40 \mu \mathrm{L}$ Buffer EB (Qiagen). PCR was performed as indicated by Buenrostro et al. (2015) [14], with minor modifications: $10 \mu \mathrm{M}$ Nextera N7xx and S5xx $2.5 \mu \mathrm{L}$ each $(0.5 \mu \mathrm{M}$ for each primer) (Nextera XT Index Kit v2, Illumina), KAPA HiFi HotStart ReadyMix (KAPA Biosystems) $25 \mu \mathrm{L}$, DNA and water to a total volume of $50 \mu \mathrm{L}$, with the PCR program: $72^{\circ} \mathrm{C}, 5 \mathrm{~min} ; 98^{\circ} \mathrm{C}, 3 \mathrm{~min} ;\left(98^{\circ} \mathrm{C} 10 \mathrm{~s}\right.$, $\left.63^{\circ} \mathrm{C} 30 \mathrm{~s}, 72^{\circ} \mathrm{C} 1 \mathrm{~min}\right) 5$ cycles; $72^{\circ} \mathrm{C} 1 \mathrm{~min}, 4^{\circ} \mathrm{C} \infty$. Beads were size-selected by $1.0 \times$ using AMPure XP beads (Beckman Coulter). Additional cycles were calculated by real-time qPCR. The final $50 \mu \mathrm{L}$ PCR product was size selected by removing large DNA fragments purified by the $0.45 \times(22.5 \mu \mathrm{L})$ concentration of AMPure $\mathrm{XP}$ beads; the DNA fragments were collected using a $1.0 \times$ final $(50 \mu \mathrm{L}$ beads) concentration of AMPure XP beads. Size selection was repeated and DNA fragments were eluted in $13 \mu \mathrm{L}$ of Buffer EB (Qiagen). 
Concentrations were measured with $1 \mu \mathrm{L}$ sample volumes (Qubit 3.0 Fluorometer) and the remaining sample was used for Tape Station analysis (Agilent Technologies) and sequencing with NextSeq 500 (Illumina).

\section{CUT\&RUN and library preparation}

CUT\&RUN analyses of H3K4me3 and CTCF localization were performed with 80,000 and $1 \times 10^{6}$ HEK293T cells, respectively, by following the CUT\&RUN protocol $[11,16]$ with minor modifications. Briefly, cells were centrifuged at $600 \times g$ for $3 \mathrm{~min}$ with wash buffer. Antibody incubation was performed for $2 \mathrm{~h}$ with the $0.02 \%$ digitonin-containing antibody buffer, which contained either $1 \mu \mathrm{g}$ anti-H3K4me3 (Abcam; ab8580) or $1 \mu \mathrm{g}$ anti-CTCF antibody (Abcam; ab70303) as described above. Cells were washed and resuspended with $0.02 \%$ digitonin/ wash buffer by centrifugation at $500 \times g$ for $5 \mathrm{~min}$ at $4{ }^{\circ} \mathrm{C}$. After the addition of pA-MNase as described above, CUT\&RUN was performed for $30 \mathrm{~min}$ on a chilled ice block and stopped by adding $2 \times$ stop buffer $(4.2 \mathrm{ml}$ water, $340 \mu \mathrm{L} 5 \mathrm{M} \mathrm{NaCl}, 200 \mu \mathrm{L} 0.5 \mathrm{M}$ EDTA, $100 \mu \mathrm{L} 0.2 \mathrm{M}$ EGTA, $20 \mu \mathrm{L} 5 \%$ Digitonin, $25 \mu \mathrm{L}$ RNase A). The CUT\&RUN library was generated using the AccelNGS $^{\circ} 1 \mathrm{~S}$ Plus DNA Library Kit (Swift Biosciences) and $1 S$ Plus Combinatorial Dual Indexing Kit (Swift Biosciences). DNA fragments were size-selected by adding $1.0 \times$ ratio of AMPure Beads XP (Beckman Coulter). DNA was amplified using the following PCR program: $98^{\circ} \mathrm{C} 30 \mathrm{~s},\left(98^{\circ} \mathrm{C} 10 \mathrm{~s}\right.$, $\left.60^{\circ} \mathrm{C} 30 \mathrm{~s}, 68^{\circ} \mathrm{C} 60 \mathrm{~s}\right) \times 5$ cycles, $4{ }^{\circ} \mathrm{C} \infty$. Additional cycles were carried out using real-time $\mathrm{qPCR}$. The final PCR amplicon was size-selected using AMPure XP Beads as described above and sequenced using NextSeq 500 (Illumina).

\section{Bioinformatics analysis \\ Cut-C data processing}

The paired-end reads for $\mathrm{Cut}-\mathrm{C}$ were processed using HiC-Pro [12]. This pipeline includes read alignment mapping to the hg19 genome, with bowtie 2 parameter (--very-sensitive -L 30 --score-min L,-0.6,-0.2 --end-toend -reorder). All parameters, except for the ligation site and minimum cis distance threshold and duplicate removal flag, were set to default. The valid interaction produced by HiC-Pro was used for quality control. The results shown in Fig. 1f and Fig. $2 \mathrm{~d}$ are based on minimum cis distance 0 and without duplicate removal.

Loop calling was carried out using cLoops with the following parameters: -m 0 -eps 5000,10000 -minPts 2,5, 3 -p 20 -w -j -s -hic -cut 2000 -plot -max_cut [19]. We also used these called loops to analyse H3K4me3 Cut-C loop frequency with regard to genomic distances between loop ends.

\section{Cut-run data processing}

The paired-end CUT\&RUN reads were processed using TrimGalore (https://github.com/FelixKrueger/
TrimGalore) with the following parameters (-stringency 5 -paired -trim 1 -length 30 -q 0 -a CTGTCT CTTATACACATCT) to trim adaptor sequences and then aligned using Bowtie [20] with the following parameters (-m 1 reads were removed using Picard's MarkDuplicates (http://broadinstitute.github.io/picard/) with default settings. The reads mapped to the blacklist features as defined in the ENCODE project [21] were removed. Peak calling was performed using MACS2 with the following parameters (callpeak -nomodel -nolambda -keep-dup all). Feature intersections were done using BEDTools [22]. For correlation analysis, the read density within a peak was calculated using featureCounts [23].

\section{Enrichment analysis}

TSS and CTCF enrichment analysis for Cut-C and CUT\&RUN were performed using ngs.plot.r [24]. Prior to TSS analysis, the read-pairing in the alignment (BAM) file produced by HiC-Pro in the Cut-C data was decoupled. This was required to prevent the inclusion of the region caused by cross-linking.

\section{CTCF motif orientation analysis}

Initially, we obtained the genome-wide CTCF motif binding location from JASPAR 2018 UCSC motif tracks (http://jaspar.genereg.net/genome-tracks/). From HiC-Pro valid interaction data, we separated the first and second anchor locations of the interacting reads. By intersecting the CTCF binding with the anchor location, we determined the motif orientation for each interacting read. Finally, the percentage of interacting reads with converging CTCF motif orientation (+ strand on $5^{\prime}$ end and - strand motif on $3^{\prime}$ end), diverging orientation $(-/+)$, or the same orientation $(+/+$ or $-/-)$ were counted and divided by the total number of interacting reads.

\section{Additional files}

Additional file $\mathbf{1}$ Cut- $C$ library validation. $\mathbf{a}, \mathbf{b}$. DNA size of Cut- $C$ libraries were validated using Tapestation, H3K4me3 libraries (a) and CTCF library (b). (PNG 493 kb)

Additional file 2 Quality control data for all Cut- $C$ data presented in this study. (XLSX 10 kb)

Additional file 3 PETs overlap between H3K4me3 Cut-C biological replicates. (XLSX $9 \mathrm{~kb}$ )

Additional file 4 Cut-C loop frequency for $\mathrm{H} 3 \mathrm{~K} 4 \mathrm{me} 3$ with regard to genomic distances between anchors. (PNG $347 \mathrm{~kb}$ )

\section{Abbreviations}

3C: Chromosome conformation capture: ChIA-PET: Chromatin interaction analysis by paired-end tag sequencing; ChIP: Chromatin immunoprecipitation; Cut-C: Cleavage under tethered nuclease for conformational capture; MNase: Micrococcal nuclease; PET: Paired end tag 


\section{Acknowledgements}

We thank Yuya Ouchi and Ryoma Yamamoto for assistance with sequencing data analysis.

\section{Authors' contributions}

TS conceived the study and designed the experiments. MK and ET performed the experiments. EW, TS, YK, and KT analysed the data. TS, YK, and $\mathrm{KT}$ wrote the manuscript with support from all authors. All authors read and approved the final manuscript.

\section{Funding}

This work was supported by JSPS KAKENHI [grant numbers JP16H05369 (KT) and JP18K16027 (TS)]. This work was also supported by Subsidies for projects to promote the enhancement of manufacturing technology for small and medium enterprises [grant number 2952711056 (StemRIM)]. These funding bodies had no role in the design of the study; the collection, analysis, and interpretation of data; and in the writing of the manuscript

\section{Availability of data and materials}

All sequencing data used in this study are deposited at GEO (GSE125988).

\section{Ethics approval and consent to participate}

Not applicable.

\section{Consent for publication}

Not applicable.

\section{Competing interests}

K.T. is a scientific founder of StemRIM. T. S and K.T. are listed as inventors on the patent applications filed by Osaka University and StemRIM for the findings described here.

\section{Author details}

'Department of Stem Cell Therapy Science, Graduate School of Medicine, Osaka University, Suita, Osaka 5650871, Japan. ${ }^{2}$ StemRIM Co., Ltd., Ibaraki, Osaka 5670085, Japan. ${ }^{3}$ Division of Gene Therapy Science, Graduate School of Medicine, Osaka University, Suita, Osaka 5650871, Japan.

Received: 28 February 2019 Accepted: 22 July 2019

Published online: 29 July 2019

\section{References}

1. Yu M, Ren B. The three-dimensional organization of mammalian genomes. Annu Rev Cell Dev Biol. 2017;33:265-89.

2. Arzate-Mejia RG, Recillas-Targa F, Corces VG. Developing in 3D: the role of CTCF in cell differentiation. Development. 2018;145(6).

3. Dekker J, Rippe K, Dekker M, Kleckner N. Capturing chromosome conformation. Science. 2002:295:1306-11.

4. Lieberman-Aiden E, van Berkum NL, Williams L, Imakaev M, Ragoczy T, Telling A, Amit I, Lajoie BR, Sabo PJ, Dorschner MO, et al. Comprehensive mapping of long-range interactions reveals folding principles of the human genome. Science. 2009:326:289-93.

5. Fullwood MJ, Liu MH, Pan YF, Liu J, Xu H, Mohamed YB, Orlov YL, Velkov S, Ho A, Mei PH, et al. An oestrogen-receptor-alpha-bound human chromatin interactome. Nature. 2009:462:58-64.

6. Mumbach MR, Rubin AJ, Flynn RA, Dai C, Khavari PA, Greenleaf WJ, Chang HY. HiChIP: efficient and sensitive analysis of protein-directed genome architecture. Nat Methods. 2016;13:919-22.

7. Han J, Zhang Z, Wang K. 3C and 3C-based techniques: the powerful tools for spatial genome organization deciphering. Mol Cytogenet. 2018;11:21.

8. Schuijers J, Manteiga JC, Weintraub AS, Day DS, Zamudio AV, Hnisz D, Lee $\mathrm{TI}$, Young RA. Transcriptional dysregulation of MYC reveals common enhancer-docking mechanism. Cell Rep. 2018;23:349-60.

9. Kai Y, Andricovich J, Zeng Z, Zhu J, Tzatsos A, Peng W. Predicting CTCF-mediated chromatin interactions by integrating genomic and epigenomic features. Nature Commun. 2018;9:4221.

10. Hsieh TH, Weiner A, Lajoie B, Dekker J, Friedman N, Rando OJ. Mapping nucleosome resolution chromosome folding in yeast by micro-C. Cell. 2015; 162:108-19.

11. Skene PJ, Henikoff S. An efficient targeted nuclease strategy for highresolution mapping of DNA binding sites. Elife. 2017;6.
12. Servant N, Varoquaux N, Lajoie BR, Viara E, Chen CJ, Vert JP, Heard E, Dekker J, Barillot E. HiC-pro. An optimized and flexible pipeline for hi-C data processing. Genome Biol. 2015;16:259.

13. Pchelintsev NA, Adams PD, Nelson DM. Critical parameters for efficient sonication and improved chromatin immunoprecipitation of high molecular weight proteins. PLoS One. 2016;11:e0148023.

14. Buenrostro JD, Wu B, Chang HY, Greenleaf WJ. ATAC-seq: a method for assaying chromatin accessibility genome-wide. Curr Protoc Mol Biol. 2015;109:21-9.

15. Corces MR, Trevino AE, Hamilton EG, Greenside PG, Sinnott-Armstrong NA, Vesuna S, Satpathy AT, Rubin AJ, Montine KS, Wu B, et al. An improved ATAC-seq protocol reduces background and enables interrogation of frozen tissues. Nat Methods. 2017;14:959-62.

16. Skene PJ, Henikoff JG, Henikoff S. Targeted in situ genome-wide profiling with high efficiency for low cell numbers. Nature Protoc. 2018;13:1006.

17. Schmid M, Durussel T, Laemmli UK. ChIC and ChEC: genomic mapping of chromatin proteins. Mol Cell. 2004;16:147-57.

18. Hsieh T-HS, Fudenberg G, Goloborodko A, Rando OJ. Micro-C XL: assaying chromosome conformation from the nucleosome to the entire genome. Nat Methods. 2016;13:1009.

19. Cao Y, Chen X, Ai D, Chen Z, Chen G, McDermott J, Huang Y, J-DJ H. Accurate loop calling for 3D genomic data with cLoops. bioRxiv. 2018;465849. https:// doi.org/10.1101/465849.

20. Langmead B, Trapnell C, Pop M, Salzberg SL. Ultrafast and memory-efficient alignment of short DNA sequences to the human genome. Genome Biol. 2009;10:R25.

21. Consortium EP. An integrated encyclopedia of DNA elements in the human genome. Nature. 2012;489:57-74.

22. Quinlan AR, Hall IM. BEDTools: a flexible suite of utilities for comparing genomic features. Bioinformatics. 2010;26:841-2.

23. Liao Y, Smyth GK, Shi W. featureCounts: an efficient general purpose program for assigning sequence reads to genomic features. Bioinformatics. 2014;30:923-30.

24. Shen L, Shao N, Liu X, Nestler E. ngs.plot: Quick mining and visualization of next-generation sequencing data by integrating genomic databases. BMC Genomics. 2014;15:284.

\section{Publisher's Note}

Springer Nature remains neutral with regard to jurisdictional claims in published maps and institutional affiliations.

\section{Ready to submit your research? Choose BMC and benefit from:}

- fast, convenient online submission

- thorough peer review by experienced researchers in your field

- rapid publication on acceptance

- support for research data, including large and complex data types

- gold Open Access which fosters wider collaboration and increased citations

- maximum visibility for your research: over $100 \mathrm{M}$ website views per year

At BMC, research is always in progress.

Learn more biomedcentral.com/submissions 